\title{
On REE and EER Methods for Mining Corner Points on the Images
}

\author{
Muhammad Sarfraz ${ }^{1}$, Zarnawab N. K. Swati ${ }^{2}$ \\ ${ }^{1}$ Department of Information Science, Kuwait University, Adailiya Campus, Safat, Kuwait; ${ }^{2}$ Department of Computer Science and \\ Information Technology, Karakoram International University, Gilgit-Baltistan, Pakistan. \\ Email: prof.m.sarfraz@gmail.com
}

Received November 2013

\begin{abstract}
This paper reviews, implements and compares two corner detection algorithms for mining corner points on the generic shapes. These corner detectors detect corners by using combination of one rectangle $(R)$ and two ellipses (EE). These algorithms have been used with different combinations: REE and EER together with different parameter settings in their descriptions. REE and EER combinations slide along the boundary of the shape and record number of boundary points in each rectangle and ellipses. REE and EER setup represent both local and global views of the image outlines and present natural corner detection methodologies to detect and mine all true corners accurately. A comparative study demonstrates the superiority of the REE and EER over some of the existing algorithms.
\end{abstract}

\section{KEYWORDS}

\section{Mining; Corner Points; Corner Detector; Planar Curves; Images}

\section{Introduction}

Mining or extracting or detecting of corner points is useful for computer applications such as image recognition, segmentation, description, and data compression. Many authors have proposed variety of algorithms in the existing literature [3-13]. Rosenfeld and Johnston [8] use k-cosine angle measurement to find corners. Rosenfeld and Weszka [9] introduce some modifications of [8] in which the maxima of the difference between successive of the averaged k-cosine angle on a digital curve between the $\mathrm{k}$ backward and forward vector were used. Freeman and Davis [6] corner detector contains the chain scanned [8] with line sliding along the contour. The change in local curvature is measured by calculating the difference in angles between consecutive segment locations and detects corners where highest change in curve occurs. The corner detector of Beus and Tiu [3] was modification of [6] which introduces an arm limit value $\tau$ to specify the upper bound for length of moving line.

This paper reviews two corner detection algorithms $[12,13]$ using boundary-based approach. These are different from ordinary techniques [3,5,6,8-11] which detect cosine angle for change in the boundary curve. These algorithms define their settings of geometries as a rec- tangle (R) and two ellipses (EE) for global, intermediate and local views respectively. Hence the settings can be denoted as REE [13] which is different from EER which was defined in [12]. Like in [10,11], these algorithms take different scales/views (global, intermediate, local) of boundary curve with defined set of shapes (REE and EER). Different parameters, in the description of REE and EER determine global, intermediate, and local views of the boundary outline curve of an image. These combinations are sliding along the planar curve and curve points are recorded at each move. Recorded curve points at each step are used for corner selection.

In addition to reviewing REE and EER methods, the paper also makes a comparative study of four popular corner detection algorithms [3,4,6,10] for boundary of shapes presented. The proposed techniques are also useful to detect corners from generic shape boundaries with or without noise.

The organization of the paper is done as follows. Section 2 gives basic formulation of the technique as well as the design of the proposed corner detection algorithm. A comparative study, with the existing algorithms in the current literature, is made in Section 3. The paper is finally concluded in Section 4. 


\section{REE and EER Methods}

This section presents two algorithms for corner detection. The two algorithms use two ellipses and a rectangle. The algorithm in Section 2.1 uses one rectangle outside two nested ellipses, let us call it as REE. The algorithm in Section 2.2 uses one rectangle inside two nested ellipses, let us call it as EER.

\subsection{REE Approach}

This proposed technique of corner detection is based on rectangle $R_{1}$ and two ellipses $E_{1,1}$ and $E_{1,2}$ sliding along the given curve. The ellipses $E_{1,1}$ and $E_{1,2}$ are embedded in $R_{1}$ such that $R_{1} \supset E_{1,1} \supset E_{1,2}$. The geometry of the rectangle and two ellipses is shown in Figure 1. To gather information about the locality of neighboring curve points, proposed technique uses rectangle and ellipses shown having common center at $p_{i}$.

The mathematical relations of $R_{1}$ and two ellipses $E_{1,1}$ and $E_{1,2}$ is described in Equation (1). The geometric structure of Figure 1 has been adopted as follows:

- The length and width of the rectangle $R_{1}$ are considered to be the lengths $2 A_{1}$ and $2 B_{1}$ respectively.

- The semi minor axis and semi major axis of the ellipse $E_{1,1}$ are considered to be the lengths $3 A_{1} / 4$ and $B_{1}$ respectively.

- The semi minor axis and semi major axis of the ellipse $E_{1,2}$ are considered to be the lengths $3 A_{1} / 4$ and $B_{1} / 2$ respectively.

$$
\begin{aligned}
& R_{1}=2 A_{1} \times 2 B_{1} \\
& E_{1,1}=\pi \times 3 A_{1} / 4 \times B_{1} \\
& E_{1,2}=\pi \times 3 A_{1} / 4 \times B_{1} / 2 \\
& \theta=\text { slope ' } S \text { ' }
\end{aligned}
$$

The length of rectangle and semi major axes of ellipses lies in the direction of slope " $S$ ". Hence the width of rectangle and semi minor axes of ellipses lie at right angle to the slope " $S$ " of the contour with center at curve point $p_{i}$, $1 \leq i \leq n$, where $n$ is the total number of contour points. Slope of curve point at $p_{i}$ is determined along the line drawn by calculating mean of five points (including $p_{i}$ ) on both sides of $p_{i}$.

By taking boundary point $p_{i}$ as center, the direction of rectangle $R_{1}$ is adjusted along major axes of the Ellipses. Similarly, ellipses $E_{1,1}$ and $E_{1,2}$ having same center at $p_{i}$ are configured with same procedure. Thus

$$
R_{1} \supset E_{1,1} \supset E_{1,2} \text {. }
$$

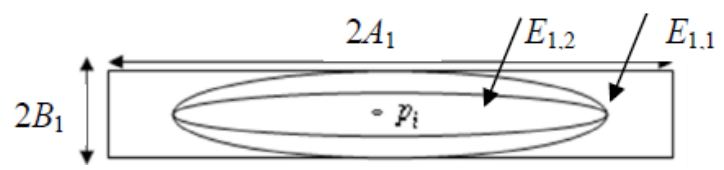

Figure 1. Geometrical structure of REE Algorithm.
Combination of rectangle and ellipses slides on given curve and number of adjacent points for rectangle and each ellipse. It is recorded from $p_{i-A}$ to $p_{i+A}$ which lie in the area $R_{1}, E_{1,1}$, and $E_{1,2}$. Let $n R_{1, i}, n E_{1,1, i}$ and $n E_{1,2, i}$ describe the total number of curve points in rectangle $R_{1}$, ellipses $E_{1,1}$, and $E_{1,2}$ respectively, with centers at $i^{\text {th }}$ boundary point. For example in Figure 2, $n R_{1, i}=23$, $n E_{1,1, i}=18$, and $n E_{1,2, i}=15$. Values of $n R_{1, i}, n E_{1,1, i}$ and $n E_{1,2, \text { i, }}$, for each boundary point, are finally used while marking the absolute positions of corner points.

Proposed algorithm adopts natural corner detection methodology by combining three levels of views. Combination of one rectangle and two ellipses represents three special views of curve points. It traces number of counts $n R_{1, i}, n E_{1,1, i}$ and $n E_{1,2, i}$, for each boundary point. It calculates sufficient information to mark the absolute corners. Rectangle $R_{1}$ represents global view of boundary points and allowing only those boundary points for which $R_{1}-E_{1,1}=0$. These contour points are represented by the set $G_{1}$ as follows:

$$
\left.\begin{array}{l}
G_{1}=\left\{P_{1, i}: n R_{i}-n E_{1, i}=0\right\} \\
\text { Or } \\
G_{1}=\left\{P_{1, i}: n R_{1, i}-n E_{1,1, i}\right\}
\end{array}\right\}
$$

Set $G_{1}$ describes wider view of a shape and does not take false corners (at contour noise/irregularities). This is demonstrated in Figure 3 which shows some snapshots with curves noise/irregularity. Center points $p_{i}$ 's in Figures 3(a) and (b) look like corners if the local view of contour is taken, but if we observed the global view (broader part of the curve), these corners are rejected as they do not qualify the Equation (2). In general, $R_{1, i}-E_{1,1, i}=0$ pointing with arrows. Curve points in Figures 3(c) and (d) are only be considered in group $G_{1}$.

Curve points lying in the set $G_{1}$ are corner points in a relative way, they can be considered as candidate corner points. These points describe common region of contour where the actual corner is located. Set $G_{1}$ describes connected points that represent a group and there is a possibility that more than one group may exist in set $G_{1}$. For each group, there is only single point which represents actual corner. The curve points in $E_{1,2, i}$ of values $n E_{1,2, i}$ less than threshold ' $\eta$ ' are calculated for every group and smallest value $n E_{1,2, i}$ is selected as a corner. If in a group the number of points in $E_{1,2, i}$ are lower than ' $\eta$ ' then it means that corner point does not exist in that group. The algorithm for REE has been devised in Figure 4.

\subsection{EER Approach}

This proposed technique of corner detection is based on rectangle $R_{2}$ and two ellipses $E_{2,1}$ and $E_{2,2}$ sliding along the given curve. The rectangle $R_{1}$ is embedded in the 


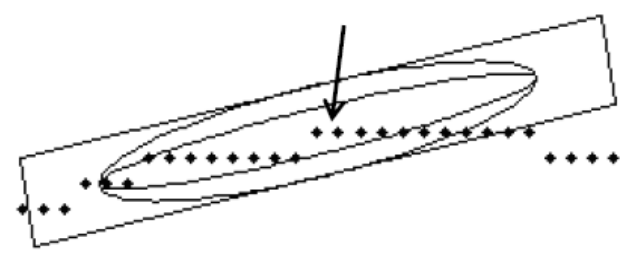

Figure 2. Snapshot of REE algorithm.

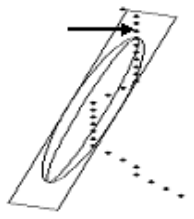

(a)

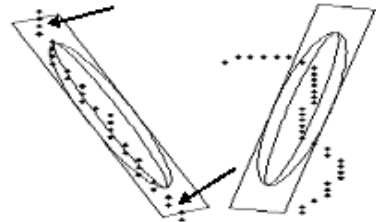

(b)

(c)

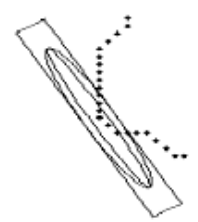

(d)
Figure 3. Some snapshots of REE Algorithm for irregular boundary. (a), (b) are not qualified by G1, and (c) is rejected due to threshold ' $\eta$ '.

For each contour point $P_{i}$

Count $n R_{1, i}, n E_{1,1, i}, n E_{1,2, i}$

End For

$G=\left\{P_{i}: n R_{1, i}, n E_{1,1, i}\right\}$

Make groups of connected points in $G$ For each group $G_{k}$

$$
\text { Corner }=\min _{n E_{2, j}}\left\{G_{k, j}: n E_{1,2, j}<\eta\right\}
$$

End For

Figure 4. Algorithm for REE.

embedded ellipses $E_{2,1}$ and $E_{2,2}$ such that $R_{1} \subset E_{2,2} \subset E_{2,1}$. The geometry of the rectangle and two ellipses is shown in Figure 5. To gather information about the locality of neighboring curve points, proposed technique uses rectangle and ellipses shown having common center at $p_{i}$.

The mathematical relations of $R_{2}$ and two ellipses $E_{2,1}$ and $E_{2,2}$ are described in Equation (3). The geometric structure of Figure 6 has been adopted as follows:

- The semi minor axis and semi major axis of the ellipse $E_{2,1}$ are considered to be the lengths $A_{2}$ and $B_{2}$ respectively.

- The semi minor axis and semi major axis of the ellipse $E_{2,2}$ are considered to be the lengths $3 A_{2} / 4$ and $B_{2}$ respectively.

- The length and width of the rectangle $R_{1}$ are considered to be the lengths $A_{2}$ and $B_{2}$ respectively.

$$
\begin{aligned}
& E_{2,1}=\pi \times A_{2} \times B_{2} \\
& E_{2,2}=\pi \times 3 A_{2} / 4 \times B_{2} \\
& R_{2}=A_{2} \times B_{2} \\
& \theta=\text { slope ' } S \text { ' }
\end{aligned}
$$

The length of rectangle and semi major axes of ellipses lies in the direction of slope " $S$ ". Hence the width of rec-

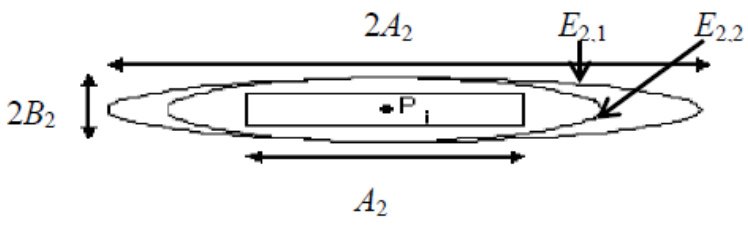

Figure 5. Geometrical structure of EER Algorithm.

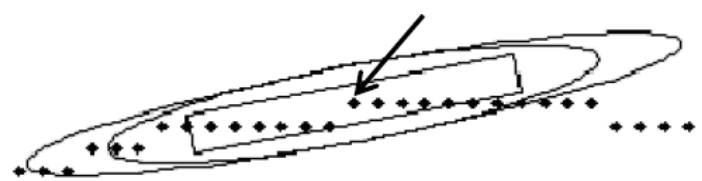

Figure 6. Snapshot of REE Algorithm.

tangle and semi minor axes of ellipses lies at right angle to the slope " $S$ " of the contour with center at curve point $p_{i}, 1 \leq i \leq n$, where $n$ is the total number of contour points. Slope of curve point at $p_{i}$ is determined along the line drawn by calculating mean of five points (including $p_{i}$ ) on both sides of $p_{i}$.

By taking boundary point $p_{i}$ as center, the direction of rectangle $R_{2}$ is adjusted along major axes of the Ellipses. Similarly, ellipses $E_{2,1}$ and $E_{2,2}$ having same center at $p_{i}$ are configured with same procedure. Thus $R_{1} \subset E_{2.2} \subset E_{2.1}$.

Combination of rectangle and ellipses slides on given curve and number of adjacent points for rectangle and each ellipse. It is recorded from $p_{i-A}$ to $p_{i+A}$ which lie in the area $R_{2}, E_{2,1}$, and $E_{2,2}$. Let $n R_{2, i}, n E_{2,1, i}$ and $n E_{2,2, i}$ describe the total number of curve points in rectangle $R_{2}$, ellipses $E_{2,1}$, and $E_{2,2}$ respectively, with centers at $i^{\text {th }}$ boundary point. For example in Figure 6, $n E_{1,1, i}=21$, $n E_{1,2, i}=18$, and $n R_{1, i}=13$. Values of $n R_{2, i}, n E_{2,1, i}$ and $n E_{2,2, \text { i, }}$, for each boundary point, are finally used while marking the absolute positions of corner points.

This algorithm adopts natural corner detection methodology (structure of ellipse is just like human eye) by combining three levels of views. Set of two ellipses and one rectangle, described above, takes three different views of contour points. Record of their counts $n R_{2, i}$, $n E_{2,1, i}$ and $n E_{2,2, i}$, for each curve point, finds enough information to locate correct corners. Ellipse $E_{2,1}$ takes broader view of digital curve and passes only those contour points for which no part of curve lies in the area $E_{2,1}$ $-E_{2,2}$. Such curve points can be described as set $G_{2}$ as follows in Equation (4):

$$
\begin{aligned}
& G_{2}=\left\{P_{2, i}: n E_{2,1, i}-n E_{2,2, i}=0\right\} \\
& \text { Or } \\
& G_{2}=\left\{P_{2, i}: n E_{2,1, i}=n E_{2,2, i}\right\}
\end{aligned}
$$

Set $G_{2}$ represents broader view of image and does not take fake corners (at curve irregularities) as discussed above. For example, Figure 7 shows some snapshots along irregular/noisy curves. Center point $p_{i}$ in Figures 7(a) and (b) appears to be corners in 


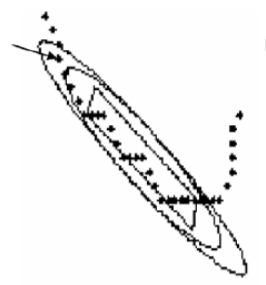

(a)

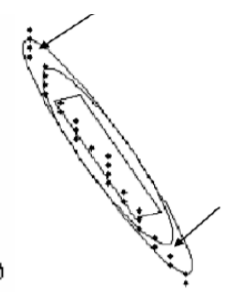

(b)

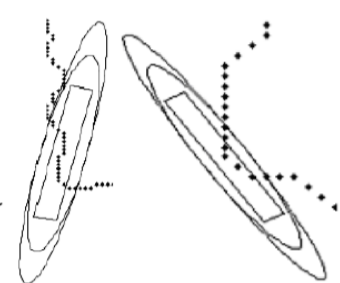

(c)

(d)
Figure 7. Some snapshots of ellipses sliding over noisy/iregular curves. Set G2 of curve does not support (a) and (b); (c) is also rejected as its nE2,2,i value is above the threshold $\eta$.

$$
\begin{aligned}
& \text { For each contour point } P_{i} \\
& \quad \text { Count } n R_{1, i}, n E_{1,1, i}, n E_{1,2, i} \\
& \text { End For } \\
& G=\left\{P_{i}: n R_{1, i}, n E_{1,1, i}\right\}
\end{aligned}
$$$$
\text { Make groups of connected points in } G
$$$$
\text { For each group } G_{k}
$$$$
\text { Corner }=\min _{n E_{2, j}}\left\{G_{k, j}: n E_{1,2, j}<\eta\right\}
$$

End For

\section{Figure 8. Algorithm for EER.}

local view (smaller part of curve is observed) but these are not the valid corners in global view (broader part of curve is observed). The EER algorithm rejected these points, because it does not satisfy the condition of Equation (4). It can be said that, some contour points of curve lie in the area $E_{2,1}-E_{2,2}$ indicated with arrows. Curve points in Figures 7(c) and (d) would only be taken in set $G_{2}$.

Curve points lying in set $G_{2}$ are not simple corners rather it shows general area of curve around the corner point as shown in Figure 7. Set $G_{2}$ describes connected points which form one group and there may be number of groups in set $G_{2}$. In each group maximum one corner point can exist. For each group, all points with $n R_{2, i}$ values below threshold " $\eta$ " are determined and the point with minimum value $n R_{2, i}$ among them is taken as a corner. Curve point in Figure 7(c) is a candidate for corner because it is part of set $G_{2}$ but does not fall below threshold $\eta$, thus it cannot be taken as a corner. Sometimes, there is no point in a group with $n E_{2,2, i}$ below $\eta$ which means corner does not exist in this group.

The EER corner detection algorithm is given in Figure 8. In this algorithm, default value of $A_{2}$ is $14 . A_{2} / 8$ and $3 A_{2} / 4$ is assigned to $B_{2}$ and $\eta$, respectively. All other parameters are relative to $A_{2}$ (Figure 5). Value of parameter $A_{2}$ depends upon the size of boundary, noise, and resolution of image. Assigned values to semi major axis, semi minor axis, length, and width of ellipses and rectangle are suitable to a certain range of size and resolution, which covers all demonstrated shapes in this paper.
These sizes were found after extensive testing on many images of similar size and resolution. The relationship between relative size of ellipses and rectangle is set (again with extensive testing) for convenience of using these parameters. The user needs to tune only one parameter i.e. $A_{2}$ instead of three. However, accuracy can improve by assigning independent sizes, but this would be at the cost of complex tuning of parameters.

\section{Experimental and Comparative Study}

To measure the accuracy of a corner detection algorithm, the absolute location of corner points must be already known. A board of 10 judges was used to mark the absolute position of corner points for standard shapes in Figure 9. Corner positions decided by majority were taken as absolute position of corners $[10,11]$. These corner positions were used to judge the accuracy of different corner detection algorithms. Detected corners by the REE and EER algorithms were shown in $[12,13]$. One can see that the corner points, detected by REE and EER, have happened to be the same as detected by human eyes. It is important to note that this evaluation is based on accuracy, localization error, noise sensitivity, transformation invariance, single response, parameter setting, and computation time. Independent tuning of three parameters $(A$, $B$ and $\eta$ ) for optimum results, once set appropriately, is enough for most of the shapes.

Different corner detection algorithms have been presented for digital boundary and their comparative study has also been described by different authors. To evaluate a corner detection algorithm, it should properly be tested on variety of shapes. Four proposed standard shapes, in Figure 9, contain noise/irregularities along the boundary curves, different types of varying curves, and angle sharpness. These types of variations are common and probable in real shape contours. These test shapes are used in this paper for comparison/evaluation of different corner detectors. Four popular algorithms, namely BT [3], CS [4], FD [6], AS [10,11] have been taken for comparison in this study. They have been compared with REE and EER algorithms presented in this paper.

A study of the six algorithms, in the light of the criteria mentioned in Section 3, has been made. Tables 1-5 show the results of the six algorithms for the proposed test shapes in Figure 9. These tables demonstrate a comparative study among the algorithms.

Accuracy results for true corner points detected by FD corner detector were lowest (60\%) for standard shapes. It is clearly indicated (in im 3 and im 4) that FD corner detector ignores some true corners. Accurate corners marked by BT corner detector is $72 \%$ were better than previous corner detector, but it also tends to miss some important corners (in im4). In case of incorrect corner detection and localization errors, the performance of BT 


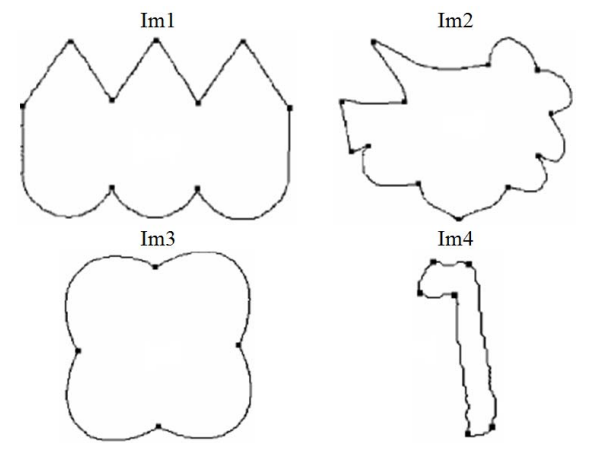

Figure 9. Test shapes with actual corner points.

Table 1. Parameter values assigned for results in Figure 9.

\begin{tabular}{|c|c|c|c|c|c|c|}
\hline & FD & BT & CS & AS & REE & EER \\
\hline im1 & $\mathrm{D}$ & $\mathrm{D}$ & $\mathrm{D}$ & $\mathrm{D}$ & $\mathrm{D}$ & $\mathrm{A}=14,1.7$ \\
\hline Im2 & D & D & D & D & $A=18$ & $\begin{array}{c}A=16 \\
B=2.42\end{array}$ \\
\hline im3 & $\begin{array}{c}S=6, \\
K=2500\end{array}$ & D & D & D & D & D \\
\hline im4 & $\begin{array}{c}K=5 \\
S=500\end{array}$ & $S=500$ & $\begin{array}{c}\operatorname{Dmin}=8 \\
\alpha \max =140\end{array}$ & D & D & $A=18$ \\
\hline
\end{tabular}

Table 2. Number of correctly detected corner points.

\begin{tabular}{ccccccc}
\hline & FD & BT & CS & AS & REE & EER \\
\hline $\mathbf{i m} 1$ & 9 & 8 & 9 & 9 & 9 & 9 \\
$\mathbf{I m} 2$ & 8 & 7 & 12 & 12 & 11 & 11 \\
$\mathbf{i m} 3$ & 3 & 4 & 4 & 4 & 4 & 4 \\
$\mathbf{i m} 4$ & 4 & 4 & 6 & 6 & 6 & 6 \\
\hline
\end{tabular}

Table 3. Number of incorrectly detected corner points in Figure 9.

\begin{tabular}{ccccccc}
\hline & FD & BT & CS & AS & REE & EER \\
\hline $\mathbf{i m 1}$ & 2 & 2 & 0 & 0 & 0 & 0 \\
$\mathbf{I m} 2$ & 3 & 3 & 9 & 5 & 1 & 0 \\
$\operatorname{im} 3$ & 5 & 0 & 0 & 0 & 0 & 0 \\
$\mathbf{i m} 4$ & 3 & 1 & 4 & 0 & 0 & 0 \\
\hline
\end{tabular}

Table 4. Correct and incorrect detected corners by each algorithm.

\begin{tabular}{ccccccc}
\hline & FD & BT & CS & AS & REE & EER \\
\hline \% Correct & 60 & 72 & 90 & 99 & 100 & 100 \\
\% Incorrect & 21 & 10 & 17 & 6 & 1 & 0 \\
\hline
\end{tabular}

Table 5. Computational time for each algorithm (in seconds).

\begin{tabular}{cccccc}
\hline Algorithm & Im2 & Im4 & Im7 & Im8 & Avg. Time \\
\hline EER & 0.9301 & 0.7443 & 1.558 & 1.7151 & 1.2369 \\
REE & 0.9567 & 0.7349 & 1.5195 & 1.7165 & 1.2319 \\
AS & 0.5631 & 0.3922 & 0.8188 & 1.0922 & 0.7166 \\
CS & 0.0106 & 0.0101 & 0.0172 & 0.0274 & 0.0163 \\
BT & 0.0508 & 0.0193 & 0.0662 & 0.0831 & 0.0549 \\
FD & 0.0253 & 0.0059 & 0.0204 & 0.0247 & 0.0191 \\
\hline
\end{tabular}

is very good and is less than $10 \%$. CS [4] gives very good results in case of correct corner detection which is $90 \%$, but its false detection rate is high and is $17 \%$ which exceeds than BT. Accurate corners marked by AS corner detector are $100 \%$, but it also detects some false corner points in im2. Performance of this algorithm is better with respect to localization errors and false detections which is $5 \%$.

A considerable improvement can be seen in REE that correctly marked $100 \%$ true corners. Lowest percentage of incorrect detection is $1 \%$ which is another big advantage of the proposed algorithm. One can hardly find localization error at any detected corner points. Performance of this algorithm must be appreciated in heavy noise conditions as well as complex shapes. No algorithm could accurately find all corners of im2, due to complex shape of the Figure. The proposed algorithm minimizes the incorrect corner(s) detection rate.

A significant improvement can also be seen in EER that correctly marked $100 \%$ true corners. One can see that no percentage of incorrect detection is found. This is another big advantage of the EER algorithm. One cannot find localization error at any detected corner points. Performance of this algorithm must be appreciated in heavy noise conditions as well as complex shapes. No algorithm could accurately find all corners of im2, due to complex shape of the figure. The proposed algorithm minimizes the incorrect corner(s) detection rate.

\section{Conclusion}

Two corner detection techniques REE and EER have been reviewed with comparative study. They do not involve curvature analysis and determination of cosine angle, hence making it very efficient. A comparative study of different corner detectors, based on proposed parameters, is given. REE and EER corner detectors have various advantages over previous corner detectors accuracy, localization error, noise sensitivity, transformation invariance, single response, parameter setting, and computation time. For the future work, it is proposed to use these algorithms for applications like vectorizing outlines of images [1] and other curve manipulation applications [2].

\section{Acknowledgements}

This work was supported by Kuwait University, Research Grant No. [WI 05/12].

\section{REFERENCES}

[1] M. Sarfraz, "Vectorizing Outlines of Generic Shapes by Cubic Spline Using Simulated Annealing,” International Journal of Computer Mathematics, Vol. 87, No. 8, 2010, pp. 1736-1751. 
http://dx.doi.org/10.1080/00207160802452519

[2] M. Sarfraz, M. Z. Hussain and M. Hussain, "Shape Preserving Curve Interpolation,” International Journal of Computer Mathematics, Vol. 89, No. 1, 2012, pp. 35-53. http://dx.doi.org/10.1080/00207160.2011.627434

[3] H. L. Beus and S. S. H. Tiu, "An Improved Corner Detection Algorithm based on Chain Coded Plane Curves," Pattern Recognition, Vol. 20, 1987, pp. 291-296. http://dx.doi.org/10.1016/0031-3203(87)90004-5

[4] D. Chetverikov and Z. Szabo, "A Simple and Efficient Algorithm for Detection of High Curvature Points in Planner Curves," Proceedings of 23rd workshop of Australian Pattern Recognition Group, Steyr, 1999, pp. 175184.

[5] E. R. Davies, "Application of the Generalized Hough Transform to Corner Detection," IEEE Proceedings of Computers and Digital Techniques, Vol. 135, No. 1, 1988, pp. 49-54.

[6] H. Freeman and L. S. Davis, "A Corner Finding Algorithm for Chain-Coded Curves," IEEE Transactions on Computers, Vol. 26, 1977, pp. 297-303. http://dx.doi.org/10.1109/TC.1977.1674825

[7] H. C. Liu and L. S. Srinath, "Corner Detection from Chain-Code,” Pattern Recognition, Vol. 23, 1990, pp. 5168. http://dx.doi.org/10.1016/0031-3203(90)90048-P

[8] A. Rosenfeld and E. Johnston, “Angle Detection on Digi- tal Curves,” IEEE Transactions on Computers, Vol. 22, 1973, pp. 875-878.

http://dx.doi.org/10.1109/TC.1973.5009188

[9] A. Rosenfeld and J. S. Weszka, “An Improved Method of Angle Detection on Digital Curves," IEEE Transactions on Computers, Vol. 24, 1975, pp. 940-941. http://dx.doi.org/10.1109/T-C.1975.224342

[10] A. Masood and M. Sarfraz, “A Novel Corner Detector Approach using Sliding Rectangles,” The Proceedings of The 4th ACS/IEEE International Conference on Computer Systems and Applications (AICCSA-06), Sharjah, 2006, pp. 621-626.

[11] A. Masood and M. Sarfraz, "Corner Detection by Sliding Rectangles along Planar Curves,” International Journal of Computers \& Graphics, Vol. 31, No. 3, 2007, pp. 440 -448. http://dx.doi.org/10.1016/j.cag.2007.01.021

[12] Z. N. K. Swati, S. Zaman, and M. Sarfraz, “A Novel Corner Detector Approach using Sliding two Ellipses and one Rectangle," The Proceedings of International Conference on Frontiers of Information Technology (FIT 2009), 16-18 December 2010, Islamabad, Article \#73.

[13] M. Sarfraz and Z. N. K. Swati, "Mining Corner Points on the Generic Shapes," Open Journal of Applied Sciences, Vol. 3, No. 1B, 2013, pp. 10-15. http://dx.doi.org/10.4236/ojapps.2013.31B003 